\title{
Coopérer pour gérer des races locales : conception, rôles et usages des instruments scientifiques de sélection
}

\author{
Julie Labatut ${ }^{1}$, Bernard Bibé ${ }^{2}$, Franck Aggeri ${ }^{3}$, Nathalie Girard ${ }^{4}$ \\ 1 Sciences de gestion, INRA, UMR1248 AGIR, 31326 Castanet-Tolosan, France \\ 2 Généticien, INRA, SAGA, 31326 Castanet-Tolosan, France \\ 3 Sciences de gestion, CGS, MinesParisTech, 75272 Paris cedex 06, France \\ 4 Sciences de gestion, INRA, UMR1248 AGIR, 31326 Castanet-Tolosan, France
}

\begin{abstract}
Julie Labatut et ses collègues, généticiens et spécialistes de sciences de gestion, appliquent un cadre d'analyse original pour rendre compte des modes de gestion des races ovines des Pyrénées-Atlantiques et comprendre comment les principes généraux de la sélection animale rencontrent les pratiques et les systèmes de valeurs traditionnels. Nous avons déjà abordé cette question dans la revue, il y a plusieurs années, avec un article de B. Vissac (NSS, 1, 4 [1993]) qui nous démontrait comment les races animales et les races locales constituaient un objet social et politique, au cœur des questions de développement agricole. Ce thème est également repris dans les contributions de J. Blanc et M. Roué (NSS, 13, 4 [2005]) et A. Lauvie et al. (NSS, 15, 2 [2007]). L'article publié dans ce numéro le confirme bien, tout en illustrant également à quel point les races et les sociétés qui les ont créées coévoluent. Il démontre, s'il en était encore besoin, le caractère hybride de ce qu'on appelle une race, relevant à la fois de la vie animale dans sa diversité biologique et des sociétés humaines dans leur conception et l'accompagnement permanent des dynamiques du monde vivant.
\end{abstract}

La Rédaction

\section{Mots-clés :}

coopération ;

races locales ;

sciences de gestion;

recherche-

intervention ;

Pyrénées-Atlantiques

\section{Keywords:}

cooperation;

local breeds;

management sciences;

intervention research;

Western-Pyrenees
Résumé - La gestion des ressources génétiques animales domestiques, et notamment des races locales, est aujourd'hui au cœur d'une actualité renouvelée qui met en question les modes de pilotage des dispositifs coopératifs sur lesquels cette gestion repose et les difficultés de coopération susceptibles de les fragiliser. À partir d'une démarche de recherche-intervention autour de la sélection des races ovines laitières locales des Pyrénées-Atlantiques, cet article identifie différentes dimensions de la coopération pour la gestion de races animales à travers l'étude de la conception, des usages et des effets inattendus des instruments scientifiques, techniques et de gestion conçus pour gérer ces races. Trois dimensions d'analyse sont développées : les tensions entre conception et usages des instruments scientifiques; les tensions entre évaluation par les instruments scientifiques et évaluation par les éleveurs dans les activités de qualification des animaux; la variété des stratégies des éleveurs sur le marché de la génétique. Cette approche par l'analyse des usages des instruments permet également de cerner des pistes d'action pour les gestionnaires de ces dispositifs.

\begin{abstract}
Cooperation for common goods production: a conceptual framework for the management of animal genetic resources. The management of domestic animal genetic resources is a topic of pressing concern given developing trends such as the liberalization of genetic resources markets, the increasing number of patents and intellectual property rights and the withdrawal of governments from the organization and control of breeding activities. Managing territorialized common goods of this nature calls for cooperative mechanisms involving a wider range of actors than so far. How to steer these mechanisms and handle crises in cooperation that can impede them, is crucial to maintaining biodiversity. This article proposes an analytical framework to analyze and facilitate cooperation in distributed breeding organizations. This framework is the result of an intervention research into breeding organizations for three local breeds of milk sheep in the Western-Pyrenees. By investigating the design and uses of scientific, technical and management instruments on which breeding activities and organization rely, we identified three dimensions to be considered in the analysis of cooperation for animal resources management: a) tensions between the design and uses of scientific instruments for genetic gain achievement; $b$ ) tensions between the evaluation of animals using scientific instruments and the evaluation of animals based on breeder know-how in qualifying animal common resources; c) diversity of farmer strategies and functioning of the market for breeding goods and services resulting from the activities conducted in the two previous dimensions (production of genetic gain, qualification of the common resources).
\end{abstract}

Auteur correspondant : J. Labatut, jlabatut@toulouse.inra.fr 


\section{Introduction}

La gestion des ressources génétiques est au cœur d'une actualité renouvelée. Elle se trouve notamment en tension entre deux évolutions principales. D'un côté, une logique néolibérale apparaît à travers la marchandisation croissante du vivant, l'augmentation des brevets et droits de propriété intellectuelle, le désengagement de l'État de l'organisation et du financement des activités de sélection ${ }^{1}$. De l'autre côté, l'émergence de logiques civiques et territoriales met en question les dispositifs de gestion de ces ressources génétiques, tout en offrant de nouvelles voies d'innovation, comme par exemple le réseau Semences paysannes (Bonneuil et al., 2006). Mais, si la dynamique de ces innovations entre science et société et, plus largement, les questions de production de connaissances pour la gestion des ressources génétiques ont fait l'objet de nombreux travaux dans le domaine végétal (Bonneuil et al., 2006; Gaudillière et Joly, 2006), elles ont été relativement peu explorées dans le cas des ressources animales, alors même que ces ressources sont au cœur d'enjeux cruciaux face aux changements actuels des activités agricoles (Joly et Paradeise, 2009).

Cet article s'intéresse plus particulièrement aux activités de sélection des ruminants qui reposent sur des dispositifs coopératifs complexes associant éleveurs, centres de sélection, organismes de développement et scientifiques autour d'un projet commun, en l'occurrence la pérennité et l'amélioration d'une population animale ${ }^{2}$. Ainsi, la nouvelle loi d'orientation agricole de janvier 2006 a mis l'accent sur la coopération volontaire pour la gestion raciale à travers la composition des organismes de sélection (comprenant de multiples participants allant des éleveurs aux collectivités territoriales). Certaines races locales ${ }^{3}$ sont particulièrement concernées par les deux évolutions précédemment exposées : intégrées à des filières de production agricole, elles sont confrontées à la concurrence de races nationales ou internationales plus productives. Mais, si cette nouvelle loi reconnaît l'importance du maintien des races locales, l'État se désengage malgré tout du financement de leur sélection. De plus, les dispositifs de sélection encadrés par la loi sur

1 Par exemple, dans le cadre du passage de la loi sur l'élevage de 1966 à la loi d'orientation agricole de 2006 en ce qui concerne les ressources génétiques animales.

${ }^{2}$ Cette recherche résulte d'une thèse réalisée à l'UMR AGIR (Agrosystèmes et développement territorial), département SAD (Sciences pour l'action et le développement) de l'Inra, à Toulouse. Elle a été financée par l'Agence nationale de la recherche (ANR) : projet O2LA (contrat ANR-09-STRA-09), et par le programme PSDR : projet INDEGICO.

3 Race locale définie comme « une race majoritairement liée, par ses origines, son lieu et son mode d'élevage, à un territoire donné » (décret du ministère de l'Agriculture et de la Pêche en application de l'article 93 de la loi d'orientation agricole du 5 janvier 2006). l'élevage de 1966 ont, malgré leur efficacité, fait l'objet de questionnements autour de la diminution de la diversité des races sélectionnées et de l'évolution de l'adaptation de ces races au territoire (Bibé et Vissac, 1979; Hansen, 2000 ; Flamant et al., 1979). Or, les ressources génétiques animales peuvent être considérées comme des biens communs, c'est-à-dire, au sens d'Ostrom (1990), des biens dont l'accès n'est pas ou peu limité, mais dont l'usage peut faire l'objet de compétition. Par exemple, dans le cas des races animales, une trop forte utilisation des meilleurs reproducteurs peut conduire à une augmentation de la consanguinité au sein d'une population animale, réduisant le potentiel de sélection future. De même, une divergence trop importante dans les objectifs de production assignés à ces ressources peut également les mettre en péril. De plus, ces races animales appartiennent à une communauté d'éleveurs qui est responsable de leur définition en tant que population présentant des caractéristiques communes et reconnaissables, et de l'appartenance d'animaux à cette population à travers la gestion de leur généalogie. L'orientation et la sélection de ces races résultent ainsi d'une action collective reposant sur différentes formes de coopération qui permettent la production de connaissances sur les animaux et leur gestion sur le long terme.

Ces formes de coopération impliquent alors des dispositifs de coordination (définition de règles collectives et de gouvernance), une variété d'acteurs participants et des connaissances partagées ou inscrites dans des règles (Raulet-Croset, 1999) qui peuvent être reconfigurées face aux enjeux actuels. Comprendre sur quoi reposent ces formes de coopération entre scientifiques et éleveurs, analyser les différents modes de production de connaissances sur les animaux et les crises éventuelles, deviennent alors essentiels pour accompagner les changements actuels autour de la gestion des ressources génétiques animales liés, par exemple, au développement des biotechnologies, à la libéralisation des marchés, ou encore à la diminution de la biodiversité.

Cet article a pour objectif d'identifier les différentes dimensions de la coopération pour la gestion des ressources génétiques par l'étude des instruments conçus et mis en œuvre pour permettre cette gestion. Pour cela, nous nous basons sur l'analyse fine $\mathrm{d}^{\prime} u$ un dispositif de sélection de races locales - celui des races ovines laitières dans les Pyrénées-Atlantiques, où le bien commun est menacé par des difficultés de coopération - et nous discutons la portée générale de ces différentes dimensions par la lecture de cas complémentaires. Notre argument est que l'analyse des instruments scientifiques et techniques sur lesquels reposent ces dispositifs, ainsi que des usages et des pratiques concrètes que les participants développent autour de ces instruments, permet à la fois de comprendre certaines des difficultés de coopération autour de ces ressources et d'offrir des pistes d'action. 


\section{Les ressources génétiques comme biens communs socialement et techniquement construits}

\section{Des «limites de l'empire technique » aux races « socialement construites »}

En France, conséquemment à la loi sur l'élevage de 1966, s'est développé un modèle de sélection d'une grande efficacité en termes de création de progrès génétique ; celui-ci repose sur une organisation intensive de la production de connaissances sur les animaux, sur l'universalité des modèles scientifiques de sélection et sur l'intervention forte de l'État (Labatut, 2009). Les limites d'un tel régime de sélection, qualifiées de « limites de l'empire technique » par Vissac (2002), en particulier les difficultés d'adaptation à des contextes variés, ont été montrées parallèlement par différents chercheurs de l'Institut national de la recherche agronomique (Inra), qu'ils soient généticiens (département Génétique animale), zootechniciens ou sociologues (département Sciences pour l'action et le développement). Dès la fin des années 1970, des solutions ont alors été imaginées pour prendre en compte une diversité de situations d'élevage, par exemple en travaillant sur l'orientation de la sélection des races ovines dans des conditions d'élevage difficiles (Flamant et al., 1979 ; Bibé et Vissac, 1979) ou dans des cas d'élevage extensif (Bougler et al., 1988). D'autres travaux ont montré que les pratiques et les rationalités des éleveurs, bien différentes des connaissances produites par la recherche, peuvent expliquer les difficultés des innovations scientifiques (Darré et al., 1993; Hubert et al., 1993; Landais, 1992; Landais et Deffontaines, 1988 ; Hubert et al., 2000). Des travaux plus spécifiques sur la sélection ont analysé la résistance au changement observée chez une partie des éleveurs dans leurs pratiques de sélection et de renouvellement du troupeau (Candau et al., 1989). D'autres encore, mobilisant les outils théoriques de l'économie des conventions, ont montré la difficulté d'intégration des formes locales de sélection, basées sur une coordination de type "domestique", au sein des schémas de sélection ${ }^{4}$ scientifique, basés sur une coordination industrielle (procédures d'évaluation objectivées dans des index génétiques) [Casabianca et Vallerand, 1994; Vallerand et al., 1994]. Plus récemment, différents travaux ont étudié les controverses auxquelles les dispositifs de sélection sont confrontés dans les modes de qualification des races locales (Lauvie, 2007 ; Lauvie et al., 2007 ; Steyaert, 2007), en combinant approches zootechnique et sociologique. En développant ainsi des analyses de l'influence des facteurs humains sur l'orientation des populations animales, des

\footnotetext{
${ }^{4}$ Nous entendons ici par «schémas de sélection » ce qui regroupe le dispositif de sélection, les objectifs de sélection et les activités de sélection permettant l'amélioration des animaux d'une population.
}

controverses et discours dont les races et leurs schémas de sélection font l'objet et des aspects sociaux des choix techniques, ces auteurs ont progressivement montré que les races animales résultent d'une construction sociale (Vissac, 2002; Audiot, 1995).

Cependant, le fonctionnement concret des dispositifs de coopération, dans leurs dimensions à la fois matérielle, cognitive et politique, reste relativement dans l'ombre, et notamment les effets des différents instruments scientifiques, techniques et organisationnels conçus pour rendre possible cette coopération. C'est pourquoi nous proposons d'analyser ces instruments conçus et utilisés pour la gestion de ces races en prenant en compte, à côté de leur dimension matérielle, le fait qu'ils sont aussi le produit d'une opération de pensée intellectuelle (doctrine d'usage, schéma abstrait) et qu'ils comportent une dimension politique (Moisdon, 2006). Notre approche vise ainsi à montrer que les races ne sont pas uniquement socialement construites, mais également conçues, produites et transformées en ressources productives grâce à plusieurs types d'instruments complémentaires : des instruments scientifiques (modèles statistiques, index génétiques), techniques (outils de mesure de la performance tels que le contrôle laitier, livres généalogiques, grilles de qualification) et organisationnels (contrats de sélection, règles collectives), pour ne citer que les principaux. L'analyse de la conception et des effets et usages inattendus de ces instruments permet de mieux comprendre les processus de coopération et les crises qui touchent cette dernière.

\section{Notre point d'entrée : analyser la coopération par les instruments de sélection}

Pour étudier ces dispositifs coopératifs, nous avons donc fait le choix méthodologique de centrer l'analyse sur le fonctionnement concret de ces derniers et sur leur instrumentation. Nous nous inspirons ici des travaux qui proposent d'étudier l'action organisée non pas à travers les discours ou les intentions des gestionnaires, mais à travers les instrumentations techniques, scientifiques ou organisationnelles qui sont mises en place pour conduire l'action collective et produire de nouvelles capacités (Moisdon, 1997; Moisdon, 2006 ; Hatchuel et Weil, 1992; Miller et Napier, 1993). L'idée est de suivre non pas les acteurs, mais les instruments, en référence aux travaux de Michel Foucault (1993) sur les instruments et les technologies de « gouvernement à distance ».

Selon ces travaux, les instruments scientifiques ou de gestion ne sont pas de simples outils, neutres, qui suivraient simplement les volontés de leurs concepteurs et de leurs usagers. Ce sont des dispositifs conçus pour " conduire les individus et les objets qu'[ils] englobent vers des finalités assignées » (Moisdon, 2006); cela est 


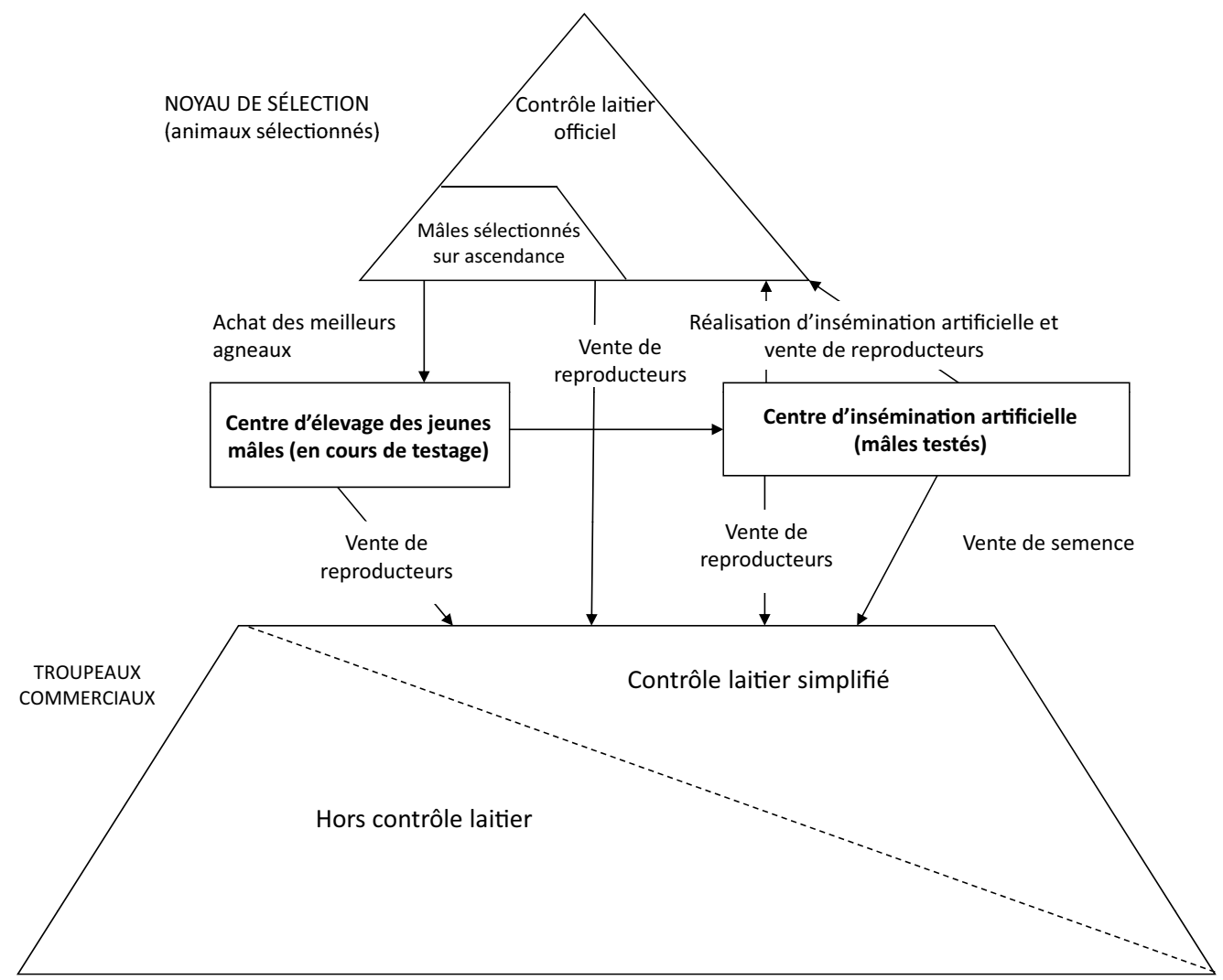

Fig. 1. Représentation simplifiée de l'organisation générale des schémas de sélection ovins laitiers (inspiré de Barillet, 1997).

particulièrement vrai pour les instruments de la sélection tels que les index de valeur génétique, qui servent à classer les animaux selon leur mérite en tant que reproducteurs et sont utilisés dans les activités économiques. Autrement dit, l'idée d'outils axiologiquement neutres n'est pas tenable dès que l'on observe les instruments de gestion en situation (Aggeri et Labatut, 2010). Notre analyse intègre donc les usages de ces instruments en situation et les effets qu'ils sont susceptibles de produire sur les comportements d'autres acteurs. Cette dimension matérielle, souvent délaissée par la littérature car considérée comme "allant de soi », n'en est pas moins essentielle pour la compréhension des dynamiques d'action collective. Ainsi, la variété des usages des instruments conçus par les scientifiques de la sélection animale et les services de développement agricole est souvent méconnue de leurs concepteurs et des gestionnaires des schémas de sélection. De même, les effets organisationnels de ces instruments sur les activités de gestion des ressources génétiques sont rarement analysés.

\section{Contexte, enjeux et méthode d'une recherche- intervention en Pyrénées-Atlantiques}

L'analyse présentée ici est issue d'une rechercheintervention menée durant trois ans autour de la sélection des races ovines laitières locales dans les PyrénéesAtlantiques, cas particulièrement pertinent pour illustrer les enjeux et les difficultés actuels de la coopération autour des ressources génétiques animales.

Dans ce département, trois races ovines laitières (Manech tête noire, Manech tête rousse, Basco-béarnaise), utilisées pour la production de fromage AOC OssauIraty, sont sélectionnées depuis les années 1975 grâce à trois schémas de sélection pilotés par un organisme de sélection $\left(e x-U P R A^{5}\right)$ et une entreprise de sélection (le Centre départemental de l'élevage ovin), et organisés sur le modèle pyramidal suivant (Fig. 1) : $20 \%$ des troupeaux de la race, en contrôle laitier officiel, produisent du progrès génétique pour le reste de la population.

Ces schémas de sélection ont été mis en place localement, avec l'aide des spécialistes de génétique animale de l'Inra, à partir des dispositifs techniques conçus et mis en œuvre dans le Rayon de Roquefort pour la sélection de la race Lacaune. Ils ont permis l'amélioration notable des performances de ces trois races selon des critères de quantité de lait (progrès génétique de +4 litres de lait par an de 1985 à 2009), cela tout en maintenant la qualité du lait (Fig. 2). Le lecteur notera un progrès génétique indéniable chez les trois races, mais plus important pour

${ }^{5}$ UPRA : Unité nationale de sélection et de promotion de la race. 


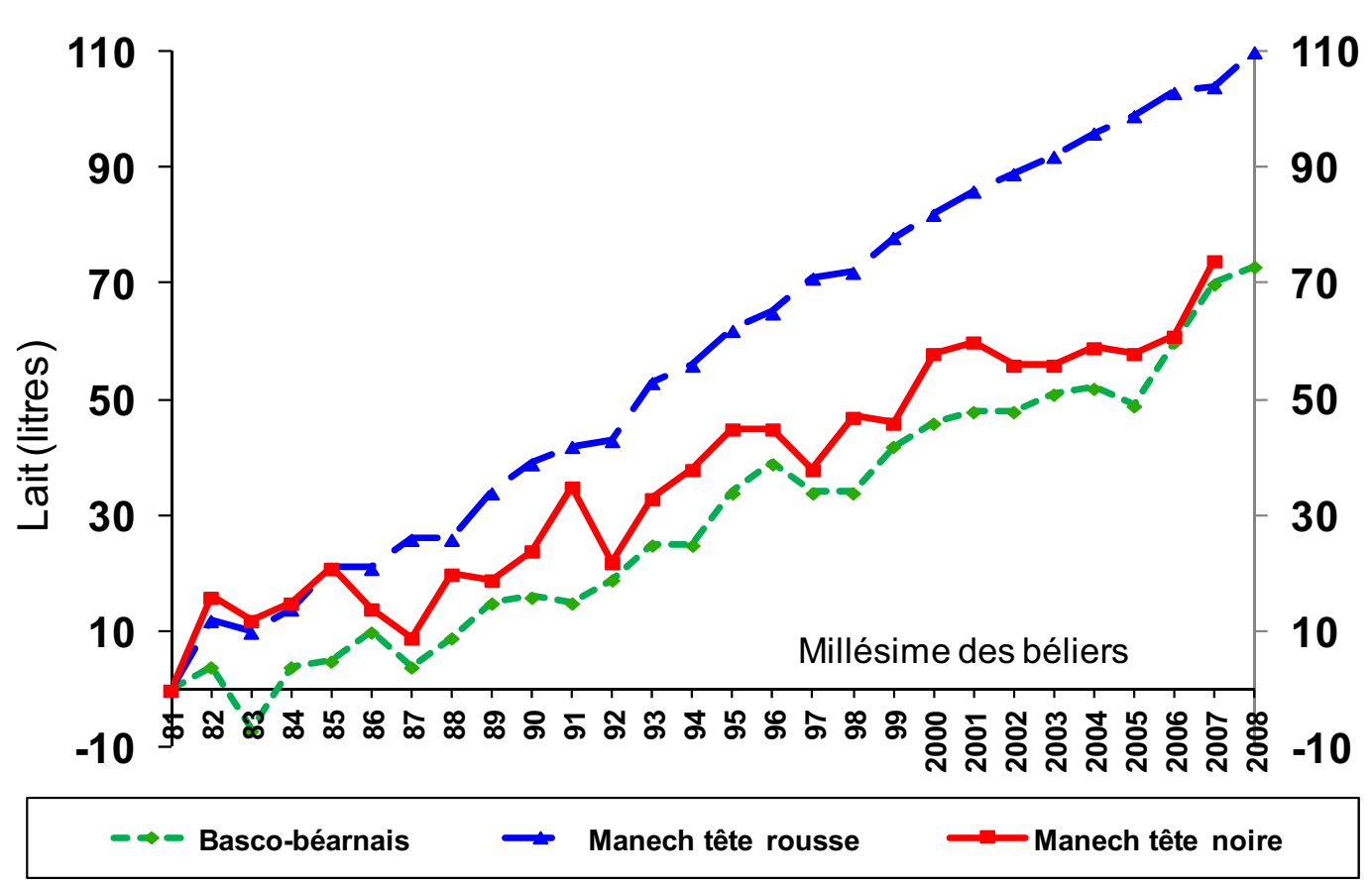

Fig. 2. Résultats des schémas de sélection des trois races pyrénéennes en termes de progrès génétique $(\delta G)$ exprimé par les moyennes des index lait, montrant l'efficacité des schémas (données Institut de l'élevage / SAGA).

la Manech tête rousse, dont le noyau de sélection est plus grand. Ces schémas ont également permis d'améliorer la résistance des animaux à la maladie de la tremblante ovine (Barillet et al., 2004).

Cependant, malgré ces améliorations génétiques, les races pyrénéennes restent confrontées à la concurrence de la race Lacaune, compte tenu, notamment, de son potentiel de production laitière supérieur et de la facilité de mécanisation de la traite avec cette race. En revanche, celle-ci est moins adaptée aux pratiques de transhumance et donc utilisée essentiellement dans le cadre de systèmes d'élevage non transhumants. De plus, malgré leur succès technique, les schémas de sélection pyrénéens sont touchés depuis quelques années par des difficultés de coopération : faible diffusion des instruments de la sélection (insémination artificielle, contrôles de performance : cf. Fig. 3), défections d'éleveurs des noyaux de sélection ${ }^{6}$, marché parallèle de reproducteurs issus par insémination artificielle, controverses sur les objectifs de sélection. Comme l'exprime l'un des animateurs des schémas de sélection, ces difficultés sont également économiques : «Notre difficulté, à l'heure actuelle, vient [non seulement] de la remise en cause des axes de sélection par certains éleveurs, mais surtout des difficultés grandissantes à financer nos actions de sélection. » (Lettre d'un des animateurs des schémas de sélection à un scientifique

\footnotetext{
${ }^{6}$ Partie de la population d'éleveurs et d'animaux engagés dans la production de progrès génétique, en contrôle laitier officiel, pour la diffusion à la base de la population.
}

afin de demander son intervention dans le cadre d'une étude prospective, le 15 juillet 2003.)

Ainsi, la diffusion des instruments scientifiques et techniques de sélection (insémination artificielle, contrôle laitier simplifié) a été beaucoup plus faible dans les Pyrénées-Atlantiques par rapport au Rayon de Roquefort ( $c f$. Tab. 1). Au sein même des noyaux de sélection, les taux d'insémination artificielle dans chaque troupeau en Pyrénées-Atlantiques restent majoritairement au niveau du minimum imposé par les contrats de sélection, tandis que, dans le Rayon de Roquefort, l'insémination artificielle a remplacé une grande partie de la monte naturelle.

Au-delà de l'engagement des généticiens de la Station d'amélioration génétique des animaux (SAGA) de 1'Inra dans la gestion des trois races ovines locales du département des Pyrénées-Atlantiques, les activités d'élevage dans ce département ont déjà fait l'objet de recherches en partenariat autour de l'agropastoralisme (Hubert et al., 2004), mais la sélection et la gestion de ces races ne constituaient pas un objet direct de ces recherches. Plus récemment, les fragilités du dispositif de sélection ont amené les généticiens de l'Inra et les responsables des schémas de sélection à s'interroger sur les raisons de ces difficultés et sur l'orientation de la sélection dans les années à venir. Après avoir construit une étude prospective sur les évolutions possibles de la filière et de la sélection des races à l'horizon 2020 - projet intitulé « Prospyr» (cf. GIS ID64, 2005) -, un partenariat original s'est constitué entre recherche en génétique (département Génétique animale de l'Inra) et recherche en sciences de gestion (UMR AGIR, 
Tableau 1. Comparaison de la plus faible diffusion du contrôle laitier simplifié et de l'insémination artificielle en PyrénéesAtlantiques par rapport au Rayon de Roquefort.

\begin{tabular}{c|c|c|c|c|c}
\hline & Effectif brebis & CLO & CLS & $\begin{array}{c}\text { \% brebis } \\
\text { en CL }\end{array}$ & $\begin{array}{c}\text { \% brebis } \\
\text { inséminées }\end{array}$ \\
\hline $\begin{array}{c}\text { Rayon } \\
\text { de Roquefort }\end{array}$ & 870000 & $\begin{array}{c}173568 \\
(376 \text { cheptels })\end{array}$ & $\begin{array}{c}522099 \\
(1322 \text { cheptels })\end{array}$ & 80 & 79,9 \\
\hline $\begin{array}{c}\text { Pyrénées- } \\
\text { Atlantiques }\end{array}$ & 475000 & $\begin{array}{c}107494 \\
(333 \text { cheptels })\end{array}$ & $\begin{array}{c}364 \\
(104 \text { cheptels })\end{array}$ & 30,4 \\
\hline
\end{tabular}

CLO : contrôle laitier officiel ; CLS : contrôle laitier simplifié ; CL : contrôle laitier.

département SAD) de l'Inra et laboratoire CGS (Centre de gestion scientifique) de l'École des mines de Paris. L'objectif était de mieux comprendre l'origine et la nature des problèmes de coopération au sein du dispositif de sélection pyrénéen, mais aussi d'aider les généticiens à mieux connaître les usages des instruments qu'ils conçoivent et les raisons de la remise en question des connaissances scientifiques. Ce sont donc les trois principaux instruments permettant la production de connaissances sur les animaux et leur sélection (contrôle laitier, insémination artificielle et index génétiques) que nous avons choisi d'analyser, afin d'identifier leur rôle dans la coopération autour des ressources génétiques animales. Nous avons ainsi identifié que les problèmes de coopération ne peuvent s'expliquer par une raison unique (un désaccord sur le standard de la race ou sur les objectifs de sélection), mais ont de multiples dimensions : (i) des tensions peuvent apparaître entre conception et usages de ces instruments dans la production du progrès génétique; (ii) ceux-ci ne suffisent pas à définir les races et l'intégration des connaissances des éleveurs dans la qualification des animaux peut faire l'objet de tensions ; (iii) ils sont plus ou moins diffusés sur le marché de la génétique du fait de la multiplicité des stratégies des éleveurs en termes de sélection. Cela nous a conduits à définir un cadre d'analyse des dispositifs coopératifs de sélection selon ces trois dimensions.

L'enjeu de cette collaboration était également de proposer des pistes d'action concrètes pour favoriser la conception d'instruments plus adaptés aux pratiques locales. Pour cela, une démarche de recherche-intervention (Hatchuel, 2000) a été menée, dans laquelle «le chercheurintervenant et les acteurs avec lesquels il travaille forment un groupe d'acteurs engagés collectivement dans un processus d'apprentissage » (David, 2000). L'objectif de ce partenariat était de relier « les faits de nature et les faits de société » (Hubert et Bonnemaire, 2000) et de favoriser les apprentissages croisés (Hatchuel, 1994) au sein des collectifs gestionnaires de ces ressources génétiques, en leur donnant les moyens de reformuler les problèmes. En effet, les tensions observées dans les Pyrénées-Atlantiques étaient parfois expliquées dans les discours des acteurs engagés dans ces actions collectives de sélection selon des catégories préconçues de résistance au changement de la part des éleveurs, de spécificité culturelle locale, de refus politique de suivre le modèle dominant. L'objectif de la collaboration entre sciences de gestion et génétiques était alors de revisiter collectivement ces discours en accédant au fonctionnement concret de ces dispositifs humains et techniques et aux pratiques effectivement mises en œuvre par les participants, ce que permet l'approche par les instruments. Celle-ci, en retour, nécessitait pour les chercheurs en sciences de gestion un dialogue avec les théories génétiques, pour mieux comprendre les principes et les hypothèses sur lesquels repose la conception des instruments scientifiques étudiés. La rechercheintervention a également reposé sur l'animation d'un groupe de travail réunissant chercheurs de l'Inra et responsables du GIS pour la construction d'un outil d'aide à la décision pour la filière ovine laitière dans les PyrénéesAtlantiques, à partir des scénarios issus du projet « Prospyr » cité plus haut.

Au-delà d'une interaction quasi quotidienne avec les scientifiques de l'unité de la SAGA et des ingénieurs de l'Institut de l'élevage, notre démarche a mobilisé des sources diverses : une soixantaine d'entretiens semidirectifs ont été effectués auprès d'éleveurs, de techniciens, d'ingénieurs intervenant dans les schémas de sélection locaux, mais également des scientifiques impliqués depuis les débuts des schémas. Les entretiens auprès des éleveurs (à la fois au sein du dispositif et en dehors) ont notamment permis de déterminer la variété des stratégies de sélection et de renouvellement du troupeau qu'ils mettaient en œuvre, et de mieux comprendre dans quelle mesure ils utilisaient les instruments de la sélection et le progrès génétique créé par les schémas de sélection, et selon quelles justifications. Plus largement, nous avons cherché dans ces entretiens à identifier le comportement des éleveurs sur le marché de la génétique et des animaux, en termes de critères de choix des animaux, de pratiques de vente, de construction des prix. Nous avons également effectué un travail d'observation participante lors du suivi des activités quotidiennes du centre de sélection (CDEO), qu'il s'agisse des étapes des 
schémas de sélection (contrôle laitier, insémination artificielle, qualification) ou des réunions sur l'orientation stratégique de la sélection. Nous avons complété ce corpus de données par le recueil et l'analyse d'archives professionnelles, scientifiques et publiques, susceptibles de nous fournir des éléments historiques sur les activités de sélection : archives du CDEO, archives personnelles de généticiens impliqués dans le département, archives départementales des Pyrénées-Atlantiques. Toutes ces informations ont été confrontées à des sources complémentaires sur d'autres races ovines laitières (Lacaune, race Corse), afin d'en retirer des éléments comparatifs pour mettre en perspective les résultats obtenus à partir du cas des Pyrénées-Atlantiques.

Nous présentons dans les parties suivantes les résultats issus de l'analyse de ce corpus de données selon trois dimensions complémentaires qui permettent de mieux comprendre les problèmes de coopération constatés et de proposer des pistes d'action pour aider à les résoudre :

- les tensions entre conception et usages des instruments pour la création de progrès génétique au sein des schémas de sélection;

- les tensions entre évaluation par les instruments scientifiques et évaluation par le savoir-faire des éleveurs dans les activités de qualification des animaux ;

- la variété des stratégies des éleveurs dans la diffusion du progrès génétique.

\section{La création du progrès génétique : comment gérer les tensions entre instruments scientifiques et pratiques locales?}

Les instruments de la sélection ont été conçus dans une logique diffusionniste (Hubert et al., 2000 ; MacCown, 2001), fondée sur la séparation entre conception et diffusion. Ainsi, cette conception a reposé sur différentes hypothèses, notamment sur celle de leur efficacité quels que soient la situation d'usage et les objectifs spécifiques de chaque utilisateur, et celle de l'universalité des connaissances scientifiques sur lesquelles ils reposent. Comment expliquer alors que ces instruments se soient moins largement diffusés dans les Pyrénées-Atlantiques que dans le Rayon de Roquefort? Une meilleure compréhension des hypothèses et des projets sur lesquels se fondent ces instruments, ainsi que de la variété de leurs usages au sein des schémas de sélection, peut fournir des éléments explicatifs à ce constat. L'analyse d'archives, de documents scientifiques et des entretiens auprès d'acteurs historiques nous permet de répondre au premier point, en montrant le projet de rationalisation général auquel les instruments de la sélection répondent, tandis que l'observation des usages actuels dans le cas des PyrénéesAtlantiques nous permet de répondre au second.

En effet, selon Hatchuel et Weil (1992), les instruments ne sont pas uniquement des objets techniques et matériels neutres dont les effets seraient entièrement contrôlés, mais intègrent dans leur conception trois éléments : substrats techniques, philosophie gestionnaire et vision simplifiée des relations organisationnelles. Les substrats techniques sur lesquels ils reposent (modèles, matériel, technique) sont accompagnés d'une philosophie gestionnaire et d'une vision simplifiée des relations organisationnelles (ibid.). Dans le cas des instruments de la sélection, les substrats techniques sont divers : méthodes, règles et supports matériels du contrôle laitier, technique de l'insémination artificielle, modèles de calcul des index de valeur génétique. La philosophie gestionnaire est le «système de concepts qui désigne les objets et les objectifs formant les cibles d'une rationalisation » (ibid., p. 124). Cette philosophie gestionnaire est une notion différente des objectifs de sélection qui vont être assignés au sein de chaque schéma de sélection. Elle précise le but général donné à l'utilisation de l'instrument, même si ce n'est pas nécessairement le but poursuivi sur le terrain. Ainsi, la philosophie gestionnaire des instruments de la sélection a reposé sur un principe visant à augmenter le revenu des éleveurs en augmentant les revenus laitiers et la performance individuelle des animaux, suivant le «paradigme de l'intensification » (Allaire et Boiffin, 2004) majoritairement suivi durant les années 1960 à 1990. Ce paradigme exprime une convention de productivité orientée vers l'objectif d'accroître la performance par unité opérationnelle. En effet, l'analyse de la conception et du contenu de ces instruments montre qu'ils permettent l'évaluation de la performance de chaque animal individuellement, et cela quels que soient les objectifs assignés collectivement au niveau du dispositif de sélection. L'hypothèse sur laquelle sont basés les index génétiques synthétiques est la possibilité de décomposer la valeur génétique des animaux en sous-unités (les index de valeur génétique) que l'on cherche à améliorer en fonction des objectifs de sélection choisis (quantité de lait, qualité du lait, morphologie mammaire). La performance du troupeau, à l'échelle globale, est alors l'addition de la performance de chaque animal, à l'échelle individuelle.

Enfin, la vision simplifiée des relations organisationnelles qui est associée aux instruments définit les rôles que doivent tenir les acteurs. En effet, les projets de rationalisation sur lesquels reposent les instruments reconfigurent le rôle des acteurs dans l'action collective. Ainsi, la mise en œuvre de l'insémination artificielle, du contrôle laitier et des index génétiques dans le cadre des schémas de sélection a impliqué la mise en place d'une relation de service (Gadrey, 1994) entre une entreprise de sélection, gérant les reproducteurs collectifs et réalisant les inséminations artificielles, et parfois le contrôle laitier, les éleveurs créateurs de progrès génétique et les éleveurs utilisateurs de progrès génétique. Selon l'organisation issue de la loi sur l'élevage de 1966, le travail de production d'information sur les animaux est alors divisé 
entre les troupeaux contrôlés, les organismes de contrôle, le système national d'information et les scientifiques qui développent les modèles statistiques et réalisent le calcul des index de valeur génétique.

Le contexte institutionnel dans lequel s'inscrit l'utilisation des instruments est l'un des facteurs aisément identifiables pouvant expliquer pourquoi ces instruments n'ont pas été aussi largement diffusés dans les Pyrénées-Atlantiques que dans le Rayon de Roquefort. Ainsi la faible cohésion de la filière de production laitière dans les Pyrénées-Atlantiques par rapport à celle du Rayon de Roquefort est à prendre en compte dans l'analyse : le syndicat de l'AOC Ossau-Iraty, l'interprofession et l'entreprise de sélection sont trois structures indépendantes dans les Pyrénées-Atlantiques, chacune développant sa propre stratégie de valorisation des ressources communes (voir notamment les travaux de LambertDerkimba, 2007). À l'opposé, dans le Rayon de Roquefort, l'existence de la Confédération générale des producteurs de lait de brebis et des industriels de Roquefort, regroupant l'interprofession, l'AOC et une partie des activités de sélection, a facilité le rassemblement de l'ensemble de la filière autour d'une stratégie commune et la diffusion et l'institutionnalisation des instruments scientifiques et techniques de la sélection génétique.

Mais notre analyse des pratiques d'éleveurs au sein des schémas de sélection ou d'éleveurs ayant récemment quitté ces schémas permet de mieux comprendre les limites de l'universalité des instruments de la sélection. Pour certains des éleveurs qui mettent l'utilisation des estives au cœur de leur stratégie de production, les «substrats techniques » des instruments de la génétique peuvent entrer en conflit avec leurs pratiques. En estive, il est difficile de contrôler la performance des animaux et de mettre en œuvre l'insémination artificielle. La traite s'y fait majoritairement à la main, ce qui rend le contrôle laitier quasi impossible. De plus, certaines estives n'étant accessibles qu'après plusieurs heures de marche, l'insémination artificielle en semence fraîche ne peut y être réalisée, comme c'est le cas pour les ovins en France; par ailleurs, il y a souvent peu d'équipements permettant de séparer les animaux. Ainsi, comme l'ont pointé les généticiens dès le début des schémas de sélection dans les Pyrénées-Atlantiques, « le contrôle laitier n'est réalisé, pour des raisons matérielles, qu'en plaine et, de ce fait, production et durée de traite sont donc sous-estimées pour environ $60 \%$ des adhérents qui pratiquent la transhumance » (Barillet et Flamant, 1977, p. 24). Cette contrainte amène alors à s'interroger sur la prise en compte de l'environnement dans les dispositifs de sélection. En effet, dans le calcul des index génétiques, l'interaction génotype-milieu est considérée comme suffisamment faible pour être négligée. On estime que l'adéquation entre les animaux sélectionnés et leur environnement est assurée par leur sélection «en ferme », c'est- à-dire directement dans leur milieu d'élevage. Or, dans les milieux fortement contraignants comme les estives, d'autant plus lorsque les performances des animaux n'y sont pas mesurées, cette interaction peut avoir une importance. Dans ce domaine, les éleveurs expriment des questionnements en termes de « rusticité » des animaux sélectionnés, de meilleure intégration de leurs connaissances et de représentation des pratiques de transhumance au sein des noyaux de sélection.

«Alors ça prendrait certainement beaucoup plus de temps, parce que c'est un travail différent, plus subtil, avec la prise en compte de l'éleveur plus importante, que uniquement contrôler l'éprouvette avec quelqu'un d'extérieur. [...] Parce que nous, on a du senti d'éleveur, mais comment on le traduit en critères, et quels moyens on se donne pour pouvoir effectivement le mesurer et le contrôler? » (Un éleveur, 20 juin 2006.)

Au-delà de l'aspect purement technique, ces difficultés d'utilisation des instruments en estive revêtent également un caractère politique. Aujourd'hui encore, pour certains éleveurs, le fait que le contrôle laitier ne soit pas réalisé en montagne est le signe que les schémas de sélection ne favorisent pas les systèmes d'élevage transhumants :

«Au centre [de sélection], ils vont un peu en contresens du pastoralisme : ils conduisent à diminuer la traite en montagne parce qu'ils ne font pas de contrôle en montagne, et il faut faire l'agnelage plus tôt parce qu'il faut faire les IA [inséminations artificielles] en juin. Pour moi, ça va à l'encontre de la défense du fromage de montagne. » (Un éleveur en estive, 19 juillet 2007.)

De même, la philosophie gestionnaire associée aux instruments de la sélection peut entrer en conflit avec les stratégies individuelles des éleveurs. Ainsi, certains ne recherchent pas en premier lieu la performance individuelle de leurs animaux, mais plutôt l'économie globale de leur troupeau, étant donné que l'estive leur offre une ressource alimentaire bon marché :

«Et je préfère autant des bêtes moyennes, mais qui sont régulières et qui durent dans le temps, plutôt qu'une bête performante qui va me faire des performances une année et qui l'année d'après est tellement fragile qu'elle va claquer. » (Un éleveur transhumant, 20 juin 2006.)

Enfin, en ce qui concerne les relations organisationnelles, la séparation entre éleveurs et troupeaux créateurs de progrès génétique et éleveurs et troupeaux utilisateurs est difficile à mettre en place. En effet, dans les estives basques, les troupeaux sont souvent gérés collectivement. La mise en œuvre des accouplements raisonnés, nécessaire à la sélection collective, y est rendue beaucoup plus difficile. Le départ en estive doit alors se faire dans la plupart des cas après la période d'accouplements. De même, l'influence des copropriétaires d'une estive collective joue également un rôle dans les pratiques des 
éleveurs et peut conduire à des usages inattendus des instruments. Certains éleveurs au contrôle laitier officiel choisissent ainsi délibérément de garder des béliers à bas niveau d'index, mais de bonne qualité esthétique, afin de ne pas s'attirer les critiques de leurs voisins qui privilégient l'aspect morphologique et esthétique des animaux plutôt que la valeur de leurs index.

Ces usages inattendus des instruments scientifiques, lorsque ceux-ci sont mis «en situation », révèlent en quoi ils ne sont pas neutres dans les processus de conception des ressources génétiques. Par les hypothèses implicites qu'ils intègrent et véhiculent, ils orientent de façon parfois inattendue ces processus et peuvent fragiliser la coopération. Ces exemples offrent des pistes d'action en termes de conception des instruments scientifiques et techniques. Par exemple, un travail spécifique sur la question de l'interaction génotype-milieu et l'analyse de son importance réelle dans un environnement tel que celui des élevages transhumants des Pyrénées-Atlantiques constituent une voie à explorer pour favoriser l'adéquation entre les instruments de la sélection et les spécificités locales.

Une deuxième dimension de l'action collective de sélection est importante pour comprendre les difficultés de coopération. En effet, dans le cas des espèces où il existe un lien fort entre éleveurs et animaux, et où la notion de race comporte de fortes dimensions identitaires, les instruments scientifiques ne suffisent pas à qualifier les animaux à sélectionner : la participation des éleveurs est nécessaire pour définir quels animaux appartiennent à la race et s'assurer de leur adéquation avec le «standard » de celle-ci. Ces activités de qualification, non plus par les instruments scientifiques de la génétique, mais par «l'œil » des éleveurs, sont également un lieu où la coopération peut être remise en question.

\section{Qualifier les animaux d'une race : comment gérer le couplage entre instruments scientifiques et connaissances des éleveurs?}

La qualification des animaux selon des critères d'appartenance à une race (critères morphologiques, esthétiques, etc.), avant même l'évaluation de leur potentiel génétique, est une dimension essentielle des activités de sélection (Vallerand et al., 1994). Plus largement, toute activité collective implique un accord sur l'objet de la coopération, c'est-à-dire la qualification des biens et des services produits. Pourtant, cette dimension de la qualification des animaux, lorsqu'elle concerne des caractères autres que directement productifs ou ayant une valeur économique ou zootechnique directe (mamelle, conformation...), est souvent considérée par les gestionnaires des schémas de sélection et les scientifiques comme mineure, voire contreproductive. Or, elle peut être un élément central pour le maintien de la coopération. L'analyse des différents types d'instruments que

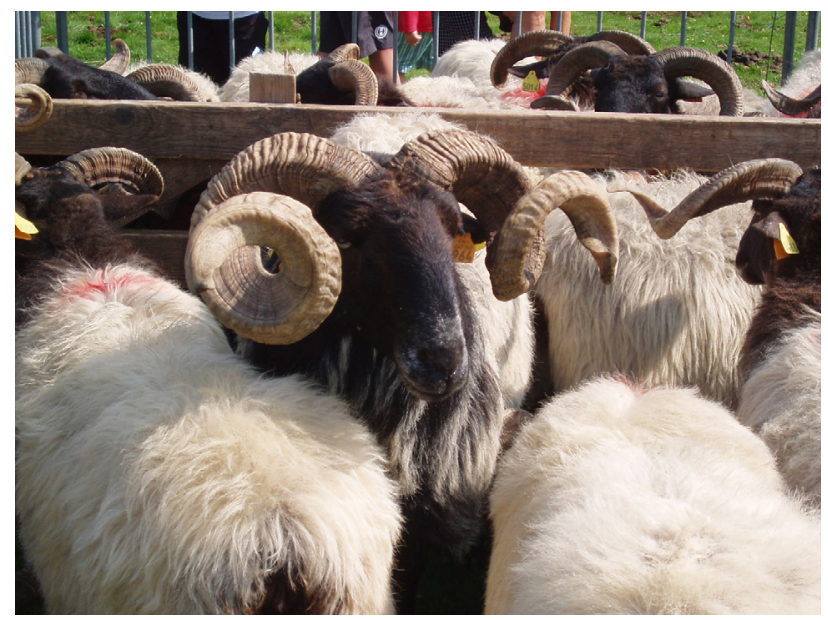

Photo. Bélier de race Manech tête noire lors d'un concours d'animaux. (Photo : J. Labatut.)

cette activité fait intervenir, et de la place des instruments scientifiques dans celle-ci, permet de mieux comprendre les tensions entre différents modes de connaissance.

Dans les Pyrénées-Atlantiques, la qualification des animaux fait l'objet de controverses de longue date, conduisant certains éleveurs à refuser les animaux issus des schémas de sélection, car ils ne correspondent pas, selon eux, au standard de la race. Ces tensions sont les plus fortes dans le cas de la race Manech tête noire (Photo), mais la question concerne ou a concerné les trois races.

Deux dispositifs de qualification en tension peuvent être identifiés, qui mobilisent différentes formes d'instrumentation pour divers objectifs :

- un dispositif organisé par l'UPRA et reconnu par l'État, faisant intervenir des éleveurs issus des noyaux de sélection dans l'évaluation des critères d'appartenance des animaux des trois races destinés à être utilisés pour la création de progrès génétique, assure l'adéquation des animaux devant être enregistrés au livre généalogique avec le standard de la race ;

- un dispositif relevant de concours d'animaux, visant à classer les plus beaux spécimens d'une population selon les critères d'éleveurs juges. Non reconnus par l'État comme élément du dispositif de sélection, ces concours sont organisés dans le cadre de foires locales et mis en avant par les acteurs de la vie économique et culturelle du territoire (commerçants, restaurateurs, banques) qui participent à leur financement. Ces dispositifs font intervenir majoritairement des éleveurs hors schémas de sélection et concernent les trois races.

Dans ces deux dispositifs, les relations entre usages des instruments scientifiques et évaluation visuelle des animaux sont complètement différentes. D'un côté, dans le dispositif UPRA, les index génétiques sont donnés aux éleveurs qui qualifient les béliers du centre de sélection. 
Le choix des animaux résulte donc d'un compromis entre un potentiel génétique prometteur et la qualité morphologique et esthétique de chaque animal. De l'autre, dans le cadre des concours, les index génétiques ne sont absolument pas pris en compte. Au contraire, la capacité des éleveurs à évaluer les animaux sur leur apparence est mise en avant.

La diversité des modes de qualification d'une même race, ici la Manech tête noire, dans un territoire peut donc affecter la coopération pour sa gestion lorsque ceux-ci se développent en opposition. Cependant, ce n'est pas parce que les instruments scientifiques ne sont pas utilisés lors des concours d'animaux que cette activité ne mobilise pas d'autres formes d'instrumentation. En effet, contrairement à ce que l'on pourrait penser, la qualification collective dans le cadre des concours repose sur une formalisation des critères d'appartenance qui permet la coordination des participants à cette activité collective et le classement des animaux les uns par rapport aux autres. Ainsi, dans ces concours, une grille de notation définissant les critères morphologiques et esthétiques de qualification est utilisée. Des règles pour le choix des juges et le déroulement du concours sont mises en œuvre (anonymat, lots d'animaux, etc.). À l'inverse, dans le cas de la qualification UPRA, l'évaluation visuelle est faiblement instrumentée. Aucune grille de qualification formalisée n'est mobilisée par les éleveurs, qui font uniquement appel à leurs connaissances tacites et personnelles du standard de la race. Seule une liste de défauts existe, mais elle n'est employée que par les techniciens et ingénieurs responsables de la gestion des informations génétiques.

Dans d'autres UPRA, les critères de qualification ont été formalisés pour rationaliser et légitimer la qualification UPRA en concevant des instruments permettant d'articuler savoirs empiriques et savoirs scientifiques dans l'évaluation des animaux. Par exemple, dans le Rayon de Roquefort, une grille très précise est utilisée par les éleveurs qui participent à la qualification. Des sessions d'étalonnage sont également organisées. Dans le cas de la race ovine Corse, pour tenter de faire face aux critiques concernant le standard des animaux issus des schémas de sélection, une démarche de formalisation des critères de qualification concernant la forme des cornes des béliers a été mise en œuvre (Aragni, 2006).

Ces exemples offrent des pistes d'action pour les schémas de sélection en Pyrénées-Atlantiques : la formalisation des critères de qualification au travers d'une grille et le partage de cette grille par les éleveurs pourraient aider à favoriser la légitimité de la sélection ; de même, la rédaction et la diffusion d'une définition du standard des races, ou encore la mobilisation des grilles des concours dans le cadre de la qualification UPRA, pourraient être envisagées pour tenter, si ce n'est une intégration, du moins un rapprochement des deux modes de qualification.
La question de la coopération autour des races locales ne peut se traiter uniquement par l'analyse des pratiques au sein des noyaux de sélection pour la création de progrès génétique. En effet, créer du progrès génétique ne suffit pas à assurer son utilisation par les éleveurs hors des schémas de sélection. Or, la diffusion du progrès génétique ainsi créé est également une dimension importante de ce travail de coopération, étant donné la visée « commune » des schémas de sélection - ceux-ci devant permettre l'amélioration d'une race dans son ensemble. La connaissance et la compréhension des pratiques et stratégies des éleveurs hors des schémas de sélection, de leur utilisation ou non du progrès génétique créé par ces schémas et des échanges de gré à gré de reproducteurs constituent un élément important pour l'analyse des dispositifs de sélection animale.

\section{Construction et fonctionnement des marchés du progrès génétique : gérer la diversité des stratégies des utilisateurs?}

Pour des raisons techniques, le modèle économique théorique des schémas de sélection ovins implique une division du travail entre une fraction des éleveurs (représentant $20 \%$ des animaux, le noyau de sélection) qui créent du progrès génétique pour eux-mêmes et l'ensemble des utilisateurs (qui détiennent les $80 \%$ restants). La vente de doses de semence sélectionnée hors des noyaux de sélection est censée assurer un retour sur investissement des schémas de sélection. Elle doit également permettre une diffusion sécurisée du progrès génétique, à la fois en termes sanitaires et de garantie d'un niveau génétique par les index qui accompagnent chaque dose d'insémination. Cette diffusion a été jusqu'à présent largement considérée par les gestionnaires des schémas de sélection et les organismes de recherche et développement comme allant de soi, tant que l'équilibre économique des dispositifs de sélection était assuré par l'État et que ces schémas garantissaient la création d'un progrès génétique jugé « efficace » (c'est-à-dire permettant l'augmentation des performances des animaux). La diffusion du progrès génétique est donc un aspect relativement peu connu de la coopération, notamment les échanges et les ventes de reproducteurs de gré à gré, part la plus importante de la diffusion dans les cas où l'insémination artificielle est peu utilisée (cas des races ovines laitières pyrénéennes et corse, mais aussi de la majorité des races à viande ovines et des races caprines). Or, dans le contexte actuel de désengagement de l'État dans le financement des activités de sélection animale (nouvelle loi d'orientation agricole de 2006), la rentabilité des dispositifs de sélection devient une forte préoccupation pour les gestionnaires.

Dans le cas des Pyrénées-Atlantiques, l'analyse du fonctionnement du marché de la génétique et du rôle des 
Tableau 2. Récapitulatif des prix identifiés lors des entretiens sur le marché des reproducteurs (2007).

\begin{tabular}{c|c|c|c|c}
\hline & \multicolumn{2}{|c|}{$\begin{array}{c}\text { Animaux produits au sein } \\
\text { des schémas (CLO) }\end{array}$} & $\begin{array}{c}\text { Animaux hors } \\
\text { contrôle laitier }\end{array}$ & $\begin{array}{c}\text { Animaux hors contrôle laitier } \\
\text { de race Manech tête noire }\end{array}$ \\
\cline { 2 - 3 } & Insémination artificielle & Monte naturelle & & $150 €$ \\
\hline Agneau & 125 à $200 €$ & 70 à $100 €$ & & 700 à $1000 €$ \\
\hline Bélier & 120 à $400 €$ & & 150 à $350 €$ & \\
\hline
\end{tabular}

CLO : contrôle laitier officiel.

instruments dans celui-ci fait apparaître des éléments inattendus permettant de mieux comprendre les problèmes de coopération. Les modes de qualification des ressources génétiques précédemment identifiés se retrouvent au niveau du fonctionnement des marchés, où plusieurs paradoxes peuvent être repérés. Tandis que de nombreux éleveurs font part d'une pénurie de reproducteurs à la fois mâles et femelles («C'est introuvable, les agnelles tête rousse ici, on achèterait 30 brebis, mais on ne peut pas trouver, en plus avec les maladies qu'il y a en ce moment. . . » : entretien, 19 juillet 2007) les encourageant à se fournir notamment en race Lacaune, dont les reproducteurs sont disponibles en plus grand nombre, le stock de reproducteurs mâles commercialisés par le centre de sélection n'est que partiellement écoulé. De nombreux éleveurs préfèrent en effet se fournir auprès de leurs collègues éleveurs plutôt que de participer aux ventes collectives du centre de sélection, alors que les reproducteurs vendus par celui-ci sont censés avoir les meilleures garanties en termes de potentiel génétique, puisqu'ils sont accompagnés des index génétiques de leurs parents. Par ailleurs, comme l'indique le tableau 2, les reproducteurs les plus chers sont vendus en dehors des schémas de sélection, parfois à des prix deux fois plus élevés que les reproducteurs issus des schémas. Ils sont vendus principalement pour leur grande qualité esthétique. Dans ces marchés, la construction des prix ne peut s'expliquer uniquement par le registre de l'ordre scientifique ou de l'ordre marchand : esthétique des animaux, réseaux sociaux, mais également proximité des pratiques entre acheteurs et vendeurs vont rentrer en jeu dans les choix de lieux d'achat.

Cependant, même si les échanges de gré à gré sont majoritaires, il était nécessaire de dépasser les discours de certains groupes d'éleveurs refusant le travail de ces schémas. Quelle est la diffusion effective du progrès génétique hors schémas de sélection? Les discours de refus se retrouvent-ils dans les pratiques concrètes des éleveurs? Notre hypothèse était que, bien que par voie indirecte, le progrès génétique issu des schémas de sélection était malgré tout diffusé dans une grande partie de la population. L'étude des pratiques des éleveurs non sélectionneurs en matière d'utilisation des instruments de la sélection, de renouvellement de leur troupeau et de sélection, mais aussi de leur stratégie vis-à-vis de l'action collective des schémas de sélection (Boisseau, 2007 ; Labatut et al., 2008) nous a permis d'élaborer une typologie de six stratégies différentes de gestion des ressources génétiques dans les exploitations. Ces résultats montrent que cinq de ces six types, soit vingt-trois des vingt-six éleveurs rencontrés hors schémas de sélection, sont utilisateurs de la génétique produite par le schéma de sélection, essentiellement par le biais de la voie mâle, c'est-à-dire via l'achat de béliers de monte naturelle à des éleveurs en contrôle laitier officiel. Toutefois, ces types d'éleveurs se distinguent les uns des autres par l'intensité avec laquelle ils utilisent la génétique, par les moyens utilisés (insémination artificielle ou monte naturelle) et par des stratégies intégrant de façon différente la transhumance, la production fromagère, la valorisation du territoire.

Ce type d'enquête apporte donc aux gestionnaires des schémas de sélection une meilleure connaissance de la variété des stratégies des éleveurs hors schémas pour le renouvellement de leur troupeau; ce qui leur permet de mieux cerner l'impact réel des schémas de sélection et d'adapter l'offre de progrès génétique à cette diversité (par exemple, en proposant différents groupes de reproducteurs pour l'insémination artificielle en fonction de stratégies orientées vers la production fermière ou non).

\section{Discussion : vers un cadre d'analyse des dispositifs de coopération pour la gestion des races locales}

L'étude que nous venons de présenter s'appuie sur un cas particulier, celui des races locales dans les PyrénéesAtlantiques, pour identifier différentes dimensions de la coopération pour la gestion de races locales. Elle nous permet de construire un cadre d'analyse des dispositifs de sélection dont nous montrons ici la généricité en faisant appel à d'autres cas. Ce cadre comporte trois dimensions (Fig. 3) : la production des biens et services de la sélection (progrès génétique, insémination artificielle, reproducteurs); la qualification des races; la diffusion de ces biens et services. Tandis que de nombreux travaux se sont intéressés soit à la qualification et aux tensions autour de la définition des races, soit aux divergences dans les objectifs de sélection, nous montrons ici que d'autres dimensions doivent être prises en compte pour mieux comprendre la diversité des problèmes de coopération, 


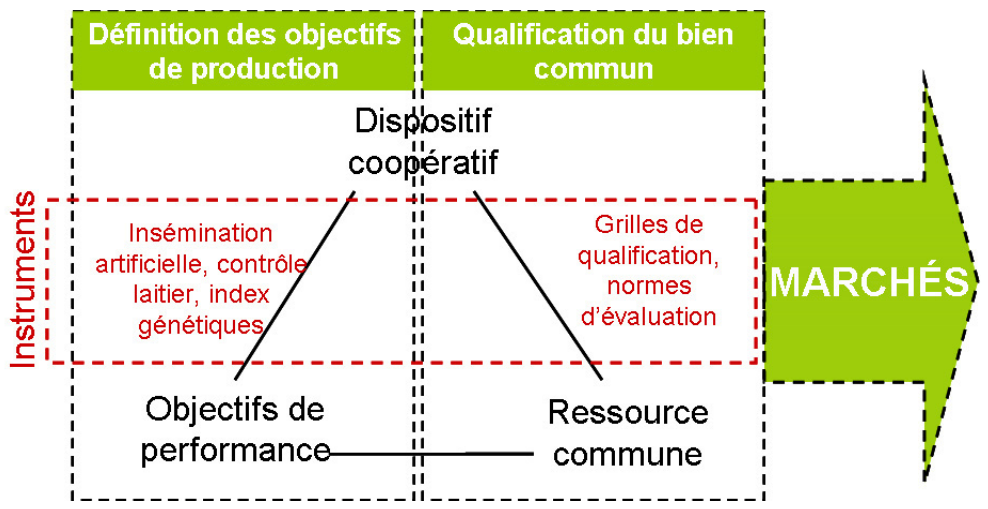

Fig. 3. Proposition de cadre d'analyse indiquant les différentes dimensions de la coopération à prendre en compte dans la gestion des ressources génétiques animales.

notamment dans le fonctionnement concret des schémas, au travers de ces trois descripteurs.

Ainsi, les facteurs favorisant ou limitant les processus de coopération se retrouvent dans d'autres dispositifs à la lumière des trois dimensions analytiques proposées ici. Concernant la dimension de création du progrès génétique, le cas de la race Lacaune illustre de quelle façon le «modèle Lacaune » (Bardini, 1991) a pu s'institutionnaliser et reposer sur une coopération forte : l'intégration de la filière a permis la définition d'objectifs et de stratégies alignés derrière la philosophie managériale des instruments de la sélection (l'ensemble des éleveurs livre à un faible nombre d'industriels, tous engagés dans la production de fromage $\mathrm{AOC}$ ) ; l'homogénéité des systèmes d'élevage et la gestion individuelle des troupeaux ont facilité l'utilisation des instruments de la sélection au sein des noyaux de sélection; des instruments d'incitation à la coopération ont été mis en place pour accompagner les instruments scientifiques et techniques (systèmes de compensation des risques liés au testage des animaux, par exemple). Dans le cas des races bovines utilisées pour la production de Beaufort (Mustard, 1998 ; LambertDerkimba, 2007), la construction d'une stratégie de filière de production de fromage d'alpage et le développement de machines à traire portatives ont favorisé l'utilisation des instruments de la sélection dans des conditions d'élevages transhumants. En Grande-Bretagne, des auteurs ont montré le rôle des instruments de la sélection dans l'évolution des rapports homme-animal, la reconstruction de communautés de pratiques et d'identités sociales (Morris et Holloway, 2009).

La deuxième dimension, celle de la qualification des animaux par les éleveurs, a été également mise en avant comme source de fragilité de la coopération dans différents dispositifs (Lauvie et al., 2007), sans que soit spécifiquement étudié le rôle des instruments dans ces activités. Certaines études révèlent, au contraire, comment ces activités de qualification ont pu être légitimées, grâce notamment à la formalisation de critères et d'instruments de qualification très précis; nous l'avons vu pour la race Lacaune ou la race Corse (Aragni, 2006), mais c'est aussi le cas pour la race porcine corse (Casabianca et al., 2000). D'autres travaux concernant les bovins ont mis en avant l'importance de ce que Grasseni (2005 et 2007) appelle «skilled vision» au sein des rapports entre homme et animal, dans un contexte de standardisation des pratiques de sélection.

La troisième dimension, celle des marchés de la génétique et de la diffusion du progrès génétique, ne peut être étudiée simplement en termes d'indicateurs tels que ceux couramment utilisés, par exemple la taille optimale du noyau de sélection par rapport à l'ensemble de la race ou la modélisation du décalage entre les valeurs génétiques moyennes des deux parties de la population (Vallerand et Elsen, 1979). L'analyse des stratégies des vendeurs et des acheteurs, des raisons de choix entre insémination artificielle ou reproducteurs, du rôle des instruments scientifiques et des critères visuels dans la construction des prix offre une meilleure compréhension des difficultés de coopération à l'échelle de la population animale.

\section{Conclusion}

L'analyse du cas des races locales dans les PyrénéesAtlantiques offre différentes perspectives. Premièrement, elle permet de montrer que les problèmes de coopération se situent à de multiples niveaux de l'action collective et ne peuvent être expliqués uniquement par des résistances au changement ou des désaccords dans les objectifs de sélection. Pour les responsables des schémas de sélection, il semble donc aussi important de tenir compte des divergences dans les objectifs de production assignés aux races sélectionnées que des tensions entre différents modes de qualification ou encore du fonctionnement des marchés du progrès génétique. La compréhension de la construction des marchés des biens et services en génétique animale, influencés notamment par les 
dynamiques d'innovations, aussi bien scientifiques que locales, reste un champ d'étude étonnamment vierge, alors que leur gestion devient essentielle pour assurer l'équilibre des dispositifs de sélection face au désengagement financier de l'État.

Deuxièmement, au-delà d'une meilleure compréhension des facteurs qui favorisent ou limitent la coopération, l'analyse des instruments de la sélection, et notamment de leurs usages, offre des pistes managériales concernant l'intégration de la diversité de ces usages et la prise en compte des pratiques locales des utilisateurs dans la conception même des instruments.

Troisièmement, le fait d'étudier les rapports entre science et pratiques locales dans la gestion des ressources génétiques animales par le biais des effets et usages des instruments sur lesquels elle repose permet un dialogue entre disciplines - dans le cas présent, génétique animale et sciences de gestion. Approcher les rapports entre les recherches en génétique animale et les dispositifs locaux par les instruments scientifiques et techniques de la sélection rend possible un travail interdisciplinaire novateur. Les chercheurs en sciences de gestion ont ici proposé des points de vue différents sur les effets et les usages des instruments conçus par les généticiens, révélant les éléments de leur non-neutralité et permettant de dépasser l'analyse des discours. Inversement, les chercheurs en génétique ont déterminé les facteurs biologiques et techniques à prendre en compte dans l'analyse des dispositifs étudiés par les sciences de gestion. Une coévolution des pratiques et des modes de pensée de la part des chercheurs dans chacune des disciplines a pu être observée durant la recherche-intervention. Il reste à multiplier les confrontations entre ce cadre analytique et d'autres études de cas afin d'en préciser les fondements, les apports et les limites.

\section{Références}

Aggeri, F., Labatut, J., 2010. La gestion au prisme de ses instruments. Une analyse généalogique des approches théoriques fondées sur les instruments de gestion, Finance, contrôle, stratégie, 13, 3, 5-37.

Allaire, G., Boiffin, J., 2004. Innovation and development: Intensification/desintensification paradigms. Reflexion from the French experience, Sixth IFSA European symposium, Vila Real, Portugal, 3-8 April.

Aragni, C., 2006. Les Défauts de cornage chez les béliers de race Corse : approche par l'étude des pratiques d'éleveurs. Master «Qualité et valorisation des productions du Bassin méditerranéen ", Université de Corse, faculté des sciences et techniques / INRA-SAD LRDE, Corte.

Audiot, A., 1995. Races d'hier pour l'élevage de demain, Paris, INRA Éditions.

Bardini, T., 1991. Modèle technique et modernisation : le cas du lait de brebis dans le Rayon de Roquefort, 1950-1985. Doctorat en sociologie, Université Paris X, Nanterre.
Barillet, F., 1997. Genetics of milk production, in Piper, L., Ruvinsky, A. (Eds), The Genetics of Sheep, Oxon (UK) and New York, CAB International.

Barillet, F., Flamant, J.-C., 1977. Principes de la sélection laitière : application à l'espèce ovine, Pâtre, numéro spécial 246, 21-31.

Barillet, F., Palhiere, I., Astruc, J.-M., Brochard, M., Baelden, M., Aguerre, X., Fidelle, F., Arranz, J., Belloc, J.-P., Briois, M., Fregeat, G., Soulas, C., Andreoletti, O., Corbiere, F., Schelcher, F., 2004. Le programme français d'éradication de la tremblante du cheptel ovin fondé sur l'utilisation de la génétique, Encéphalopathies spongiformes transmissibles animales, INRA Productions animales, numéro hors série, 87-100.

Bibé, B., Vissac, B., 1979. Amélioration génétique et utilisation $\mathrm{du}$ territoire, in Molénat, G., Jarrige, R. (Eds), Utilisation par les ruminants des pâturages d'altitude et parcours méditerranéens, Versailles, INRA Éditions, 481-491.

Boisseau, E., 2007. Diversité des pratiques de gestion des ressources génétiques par les éleveurs ovins laitiers : vers une amélioration de la gestion collective des races locales en Pyrénées-Atlantiques. Mémoire de fin d'études d'ingénieur, ENITA, ClermontFerrand.

Bonneuil, C., Demeulenaere, É., Thomas, F., Joly, P.-B., Allaire, G., Goldringer, I., 2006. Innover autrement? La recherche face à l'avènement d'un nouveau régime de production et de régulation des savoirs en génétique végétale, in Gasselin, P., Clément, O. (Eds), Quelles variétés et semences pour des agricultures paysannes durables?, Paris, INRA, 29-51.

Bougler, J., Bibé, B., Verrier, E., 1988. Choix et sélection d'un matériel génétique animal adapté ou adaptable aux systèmes extensifs. Association française de zootechnie (AFZ), session d'hiver.

Candau, J., Gachiteguy, A., Fourquet, F., Jaureguiberry, F., 1989. Innovation et résistance au développement : la filière du lait de brebis au Pays basque (Hasparren et Baigorri), Pau, Université de Pau et des Pays de l'Adour.

Casabianca, F., Vallerand, F., 1994. Gérer les races locales d'animaux domestiques : une dialectique entre ressources génétiques et développement régional, Genetics Selection Evolution, 26, Supp. 1, S343-S357.

Casabianca, F., Poggioli, A., Rossi, J.D., Maestrini, O., 2000. L'amorce d'une gestion collective de la race porcine corse. Construire un standard et élaborer les contrôles des reproducteurs, Options méditerranéennes, Série A, 41, 23-34.

Darré, J.-P., Hubert, B., Landais, É., Lasseur, J., 1993. Raisons et pratiques. Dialogues avec un éleveur ovin, Études rurales, 131-132, 107-183.

David, A., 2000. La recherche-intervention, cadre général pour la recherche en management?, in David, A., Hatchuel, A., Laufer, R. (Eds), Les Nouvelles Fondations des sciences de gestion, Paris, Vuibert, 193-212.

Flamant, J.-C., Bibé, B., Gibon, A., Vu Tien Khang, J., 1979. Approches pour une amélioration des races locales. Notions de rusticité, in L'Amélioration génétique des espèces ovines et caprines : $5^{\text {es }}$ Journées de la recherche ovine et caprine, 5 et 6 décembre 1979, Paris, INRA/Institut technique de l'élevage ovin et caprin.

Foucault, M., 1993. Surveiller et punir, Paris, Gallimard.

Gadrey, J., 1994. Les relations de service et l'analyse du travail des agents, Sociologie du travail, 36, 3, 381-389.

Gaudillière, J.-P., Joly, P.-B., 2006. Appropriation et régulation des innovations biotechnologiques : pour une comparaison transatlantique, Sociologie du travail, 48, 330-349. 
GIS ID64, 2005. L'Élevage ovin laitier des Pyrénées-Atlantiques en 2020, Ordiarp.

Grasseni, C., 2005. Designer cows: The practice of cattle breeding between skill and standardization, Society and Animals, $13,1,33-49$.

Grasseni, C., 2007. Managing cows: An ethnography of breeding practices and uses of reproductive technology in contemporary dairy farming in Lombardy (Italy), Studies in History and Philosophy of Biological and Biomedical Sciences, 38, 488-510.

Hansen, L.-B., 2000. Consequences of selection for milk yield from a geneticist's viewpoint, Journal of Dairy Science, 83, $1145-1150$.

Hatchuel, A., 1994. Apprentissages collectifs et activités de conception, Revue française de gestion, 99, 109-120.

Hatchuel, A., 2000. Recherche, intervention et production de connaissances. Communication au symposium Recherches pour et sur le développement rural, INRA/DADP, Montpellier, 12 janvier.

Hatchuel, A., Weil, B., 1992. L'Expert et le système : gestion des savoirs et métamorphose des acteurs dans l'entreprise industrielle, Paris, Economica

Hubert, B., Bellon, S., Girard, N., Lasseur, J., 1993. Pratiques d'éleveurs et connaissances de chercheurs. Communication au $2^{e}$ Congrès européen de systémique, 5-8 octobre, AFCETCSCI-UES, Prague.

Hubert, B., Bonnemaire, J., 2000. La construction des objets dans la recherche interdisciplinaire finalisée : de nouvelles exigences pour l'évaluation, Natures Sciences Sociétés, 8, 3, 5-19.

Hubert, B., Ison, R.L., Röling, N., 2000. The "problemati-

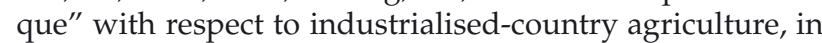
LEARN Group [Cerf, M., Gibbon, D., Hubert, B., Ison, R., Jiggins, J., Paine, M., Proost, J., Roling, N.], Cow Up a Tree: Knowing and Learning for Change in Agriculture. Case Studies from Industrialised Countries, Paris, INRA Éditions, 13-29.

Hubert, B., Moulin, C.H., Roche, B., Pluvinage, J., Deffontaines, J.-P., 2004. Quels dispositifs pour conduire des recherches en partenariat? L'intervention d'une équipe de recherche au Pays basque intérieur, Économie rurale, 279, 33-52.

Joly, P.-B., Paradeise, C., 2009. Introduction. Agriculture et alimentation : nouveaux problèmes, nouvelles questions, Sociologie du travail, 45, 1-8.

Labatut, J., 2009. Gérer des biens communs : processus de conception et régimes de coopération dans la gestion des ressources génétiques animales. Doctorat en sciences de gestion, École nationale supérieure des mines, Paris.

Labatut, J., Aguerre, X., Arranz, J.-M., Astruc, J.-M., Bibé, B., Boisseau, E., Girard, N., Thénard, V., 2008. Diversité des pratiques de gestion des ressources génétiques par des éleveurs ovins laitiers : vers une meilleure connaissance des usages des outils de sélection pour piloter la gestion collective des races locales en Pyrénées-Atlantiques, Journées Rencontres Recherches Ruminants (3R), Paris.

Lambert-Derkimba, A., 2007. Inscription des races locales dans les conditions de production des produits animaux sous AOC : enjeux et conséquences pour la gestion collective des races mobilisées. Doctorat en zootechnie, AgroParisTech, Paris.

Landais, É., 1992. Tendances actuelles des recherches sur les systèmes d'élevage : exemples des travaux du département
Systèmes agraires et développement de l'INRA, Cahiers Agricultures, 1, 1, 55-65.

Landais, É., Deffontaines, J.-P., 1988. Les pratiques des agriculteurs. Point de vue sur un courant nouveau de la recherche agronomique, Études rurales, 109, 125-158.

Lauvie, A., 2007. Gérer les populations locales à petits effectifs : approche de la diversité des dispositifs mis en œuvre. Doctorat en gestion des ressources génétiques animales, AgroParisTech, Paris.

Lauvie, A., Casabianca, F., Verrier, E., Audiot, A., Brives, H., 2007. Gestion des populations animales à petits effectifs. Accès aux dispositifs par l'analyse des controverses, Natures Sciences Sociétés, 15, 154-161.

MacCown, R.L., 2001. Learning to bridge the gap between science-based decision support and the practice of farming: Evolution in paradigms of model-based research and intervention from design to dialogue, Australian Journal of Agricultural Research, 52, 5, 549-571.

Miller, P., Napier, C., 1993. Genealogies of calculation, Accounting, Organizations and Society, 18, 7-8, 631-647.

Moisdon, J.-C., 1997. Du mode d'existence des outils de gestion, Paris, Seli Arslan.

Moisdon, J.-C., 2006. Sur la largeur des mailles du filet: savoirs incomplets et gouvernement des organisations, in Hatchuel, A., Pezet, E., Starkey, K., Lenay, O. (Eds), Gouvernement, organisation et gestion : l'héritage de Michel Foucault, Laval, Les Presses de 1'Université Laval, 135-146.

Morris, C., Holloway, L., 2009. Genetic technologies and the transformation of the geographies of UK livestock agriculture: A research agenda, Progress in Human Geography, 33, 3, 313-333.

Mustar, P., 1998. La relance du fromage de Beaufort, in Les Chercheurs et l'innovation: regards sur les pratiques de l'INRA, Paris, INRA, 84-115.

Ostrom, E., 1990. Governing the Commons: The Evolution of Institutions for Collective Action, Cambridge, Cambridge University Press.

Raulet-Croset, N., 1999. Processus de structuration et émergence de la coopération, in Froehlicher, T., Vendemini, S. (Eds), Connivences d'acteurs, contrats, coopération interentreprises et métamorphose des organisations, Nancy, Presses universitaires de Nancy, 225-240.

Steyaert, P., 2007. The Maraîchine breed: A biological object mediating various forms of knowledge, International Social Science Journal, 58, 187, 87-96.

Vallerand, F, Elsen, J.M., 1979. La diffusion du progrès génétique : étude théorique et quelques applications, in L'Amélioration génétique des espèces ovines et caprines: $5^{\text {es }}$ Journées de la recherche ovine et caprine, 5 et 6 décembre 1979, Paris, INRA/Institut technique de l'élevage ovin et caprin, 76-98.

Vallerand, F., Casabianca, F., de Sainte Marie, C., Bouche, R., 1994. D'une qualité à une autre. Conduire le changement du système de qualification des reproducteurs de race ovine corse, Études et recherches sur les systèmes agraires et le développement, 28, 157-175.

Vissac, B., 2002. Les Vaches de la République: saisons et raisons d'un chercheur citoyen, Paris, INRA.

Reçu le 29 mai 2009. Accepté le 9 septembre 2011. 